Draft Version OCtober 20, 2017

Preprint typeset using $\mathrm{LAT}_{\mathrm{E}} \mathrm{X}$ style emulateapj v. 01/23/15

\title{
GAS CONTENTS OF GALAXY GROUPS FROM THERMAL SUNYAEV-ZEL'DOVICH EFFECTS
}

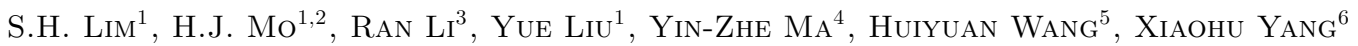 \\ Draft version October 20, 2017
}

\begin{abstract}
A matched filter technique is applied to the Planck all-sky Compton $y$-parameter map to measure the thermal Sunyaev-Zel'dovich (tSZ) effect produced by galaxy groups of different halo masses selected from large redshift surveys in the low- $z$ Universe. Reliable halo mass estimates are available for all the groups, which allows us to bin groups of similar halo masses to investigate how the tSZ effect depends on halo mass over a large mass range. Filters are simultaneously matched for all groups to minimize projection effects. We find that the integrated $y$-parameter and the hot gas content it implies are consistent with the predictions of the universal pressure profile model only for massive groups above $10^{14} \mathrm{M}_{\odot}$, but much lower than the model prediction for low-mass groups. The halo mass dependence found is in good agreement with the predictions of a set of simulations that include strong AGN feedback, but simulations including only supernova feedback significantly over predict the hot gas contents in galaxy groups. Our results suggest that hot gas in galaxy groups is either effectively ejected or in phases much below the virial temperatures of the host halos.
\end{abstract}

Subject headings: methods: statistical - galaxies: formation - galaxies: evolution - galaxies: halos.

\section{INTRODUCTION}

In the current paradigm of galaxy formation, galaxies are thought to form and evolve within dark matter halos (see Mo et al. 2010, for a review). During the formation of dark matter halos, the cosmic gas component first moves along with the dark matter, then gets shock-heated as the halos collapse, and eventually forms hot gaseous halos. In an adiabatic case, the resulting distribution of the hot gas component follows roughly that of dark matter, and the amount of the hot gas per dark matter is roughly a constant, about the universal baryon fraction of the universe. In reality, however, a myriad of other physical processes, such as radiative cooling, star formation, feedback from supernovae ( $\mathrm{SNe}$ ) and active galactic nuclei (AGN), etc, can change the hot gas content of the halos. Indeed, the hot gas fractions in low-mass halos are found to be lower than the universal baryon fraction both in observations (e.g. David et al. 2006; Gastaldello et al. 2007; Pratt et al. 2009; Sun et al. 2009) and in numerical simulations (e.g. McCarthy et al. 2010; Battaglia et al. 2013; Le Brun et al. 2014).

\footnotetext{
${ }^{1}$ Department of Astronomy, University of Massachusetts, Amherst MA 01003-9305; slim@astro.umass.edu

${ }^{2}$ Physics Department and Center for Astrophysics, Tsinghua University, Beijing 10084, China

${ }^{3}$ Key laboratory for Computational Astrophysics, National Astronomical Observatories, Chinese Academy of Sciences, Beijing, 100012, China

${ }^{4}$ School of Chemistry and Physics, University of KwaZuluNatal, Westville Campus, Private Bag X54001, Durban, 4000, South Africa; NAOC-UKZN Computational Astrophysics Centre (NUCAC), University of KwaZulu-Natal, Durban, 4000 South Africa

${ }^{5}$ Key Laboratory for Research in Galaxies and Cosmology, Department of Astronomy, University of Science and Technology of China, Hefei, Anhui 230026, China; School of Astronomy and Space Science, University of Science and Technology of China, Hefei 230026, China

${ }^{6}$ Department of Astronomy, and Tsung-Dao Lee Institute, Shanghai Jiao Tong University, Shanghai 200240, China; IFSA Collaborative Innovation Center, Shanghai Jiao Tong University, Shanghai 200240, China
}

Even in massive systems, such as rich clusters of galaxies where the total hot gas is found to be closer to the universal value, the distribution of the hot gas is found to be different from that of dark matter (e.g. Arnaud et al. 2010; Battaglia et al. 2012). However, current observational results are still uncertain, particularly for lowmass systems, and many competing theoretical models have been proposed to describe the formation and structure of gaseous halos. Clearly, an accurate determination of the hot gas content in dark matter halos is crucial for understanding galaxy formation and evolution in a way complimentary to the information provided by stars and cold gas.

The thermal Sunyaev-Zel'dovich effect (tSZ hereafter; Sunyaev \& Zeldovich 1972) provides a promising avenue to probe the hot gas in halos. As the CMB photons pass through galaxy systems, such as clusters and groups of galaxies (collectively referred to as groups of galaxies), they are scattered by the hot electrons by the inverse Compton process, producing a net energy gain in the photon gas and changing the CMB temperatures in the directions to the groups. Thus, studying the crosscorrelation of the imprints of tSZ effect on the CMB with galaxy groups allows one to probe the hot gas components in halos associated with galaxy systems. Compared to X-ray observations, the tSZ effect is less sensitive to the hot gas density, thus making it possible to explore the hot gas in the outskirts of halos and also in low-mass halos where the gas density is expected to be low.

However, extracting the tSZ signal reliably from $\mathrm{CMB}$ observations is not easy. First, the signal to be detected is usually comparable to or lower than the primary CMB, and there are also contaminations, such as Galactic emissions, dust, and point sources. As a result, individual detection and analysis of the tSZ effect are currently only possible for rich clusters of galaxies (e.g. Planck Collaboration V 2013). For low-mass groups, stacking of many systems is required to increase the signal-to-noise. Second, the beam sizes of current instruments are usually 
insufficient to resolve low-mass systems, so that assumptions about the spatial distribution of the hot gas are required. Finally, the signal from low-mass systems can be contaminated by the projections of larger halos along the same line-of-sights, and such contamination is not straightforward to eliminate.

Recently, Planck Collaboration XI (2013) used the all-sky Planck Compton parameter map and a sample of locally brightest galaxies as tracers of dark matter halos to investigate the tSZ effects produced by galaxy systems with halo masses down to $\sim 4 \times 10^{12} \mathrm{M}_{\odot}$. Remarkably, their results show that the universal pressure profile (UPP) model, in which the hot gas fraction relative to halo mass is independent of halo mass, matches their data well. This finding is in conflict with the results obtained from X-ray observations and hydrodynamic simulations where a much lower fraction is found for hot gas in low-mass systems. Using a hydrodynamic simulation, Le Brun et al. (2015) found that the universal pressure profile of Arnaud et al. (2010) adopted by Planck Collaboration XI (2013) in their matched filter method may lead to over-estimations of the integrated tSZ signal for low-mass systems. However, Greco et al. (2015) showed that adopting another popular pressure profile of Battaglia et al. (2012), instead of that of Arnaud et al. (2010), leads to differences that are well within the observational uncertainties, and so the high gas fraction found by Planck Collaboration XI (2013) cannot be explained by the adopted profile. Ma et al. (2015) crosscorrelated the Planck tSZ map with gravitational lensing map from CFHTLenS survey and found that the prediction of UPP model is $20 \%$ higher than the data. Vikram et al. (2017) cross-correlated the Planck tSZ map with the group catalog of Yang et al. (2007) and found that the two-halo terms dominate the tSZ signal for systems of $M_{200} \leq 10^{13} h^{-1} \mathrm{M}_{\odot}$, indicating that projection effect is an important issue.

In this paper, we extract the tSZ signal from the Planck all-sky observation for galaxy systems of different halo masses, using the group catalog of Lim et al. (2017). The catalog is constructed for four large redshift surveys with the use of the halo-based group finder of Yang et al. (2005, 2007). This provides the largest sample of galaxy groups in the low- $z$ universe to study the tSZ effects over a large range of galaxy systems. In particular, reliable halo mass estimates are provided for all groups, so that we can bin groups of similar masses to investigate how the tSZ effect depends on halo mass. We employ the matched filter technique (Haehnelt \& Tegmark 1996; Herranz et al. 2002; Melin et al. 2005, 2006) to extract the tSZ signal from the Planck map. In particular, we simultaneously match the filters to all galaxy systems in the catalog, so that projection effects produced by halos along the line-of-sights are properly taken care of.

The outline of this paper is as followings. We describe the observational data used in our analysis in Section 2, and our method to extract the tSZ signal in Section 3. We present our main results as well as comparisons with results from earlier studies and from numerical simulations in Section 4. Finally, we summarize and conclude in Section 5.

We adopt the cosmological parameters from the Planck observation (Planck Collaboration XIII 2016), $\left\{\Omega_{\mathrm{m}}, \Omega_{\Lambda}, h, \sigma_{8}\right\}=\{0.308,0.692,0.678,0.831\}$ through- out this paper unless specified otherwise.

\section{OBSERVATIONAL DATA}

\subsection{The Planck $y$-map}

The Planck (Tauber et al. 2010; Planck Collaboration I 2011), a space mission to measure the CMB anisotropy, is an all-sky observation in nine frequency bands ranging from 30 to $857 \mathrm{GHz}$, with angular resolutions from 31' to $5^{\prime}$. For our analysis of the thermal Sunyaev-Zel'dovich (tSZ) effects, we use the Planck NILC (Needlet Independent Linear Combination; Remazeilles et al. 2011) allsky tSZ Compton parameter map (Planck Collaboration XXII 2016), also referred to as the NILC $y$-map, which is part of the publicly released Planck 2015 data $^{7}$. The map is constructed from the full mission data set, using a combination of different frequency maps to remove the primary CMB fluctuations and to minimize contamination from foreground sources. For more details of the $y$-map construction, the readers are referred to the original paper cited above. To limit the Galactic foreground contamination, which is mainly due to thermal dust emissions, we mask the brightest $40 \%$ of the sky by applying the corresponding mask provided in the Planck 2015 data release. For contamination from extra-galactic sources, such as radio and infrared galaxies, we apply the mask provided in the same data release for point sources.

\subsection{Galaxy groups}

In order to determine the tSZ signals from halos associated with different galaxy systems, we need a well-defined group catalog that provides reliable information for both the positions and halo masses of the galaxy systems in the universe. Furthermore, since the tSZ signals are typically weak for individual groups, and since it is necessary to stack many systems to increase the signal to noise ratio, a well-defined group catalog is also needed to interpret the stacking results. In this paper we use the group catalogs given in Lim et al. (2017), which uses four redshift catalogs of galaxies (2MRS, 6dF, SDSS, and 2dF) to achieve an almost all-sky $(91 \%)$ coverage and the best depth reachable by these galaxy catalogs in each region of the sky. Groups are identified with the adaptive halobased group finder of Yang et al. (2005, 2007) with some modifications (see Lim et al. (2017) for the detail). Tests with realistic mock galaxy catalogs show that the halo masses assigned by the group finder match well the true masses, with typical scatter of $0.2-0.3$ dex. The catalogs provide two different halo mass estimates based either on the luminosities and stellar masses of member galaxies, and we use the masses based on the stellar masses. We combine 2MRS, 6dF, and SDSS to construct our sample of groups with $\log M_{500} / \mathrm{M}_{\odot} \geq 12$ for the tSZ analysis. For sky regions covered by more than one catalog, the preference is given in the order of SDSS, 6dF, and 2MRS. The sample contains a total of 471,696 galaxy systems (groups), of which 3,851 have $\log M_{500} / \mathrm{M}_{\odot} \geq$ $14,112,494$ have $13 \leq \log M_{500} / \mathrm{M}_{\odot} \leq 14$, and 240,747 have $12 \leq \log M_{500} / \overline{\mathrm{M}}_{\odot} \leq 13$. Following conventions in previous $\mathrm{SZ}$ effect analyses, we define a halo by a radius $R_{500}$, within which the mean density is 500 times the critical density at the redshift in question. The mass,

7 https://pla.esac.esa.int 


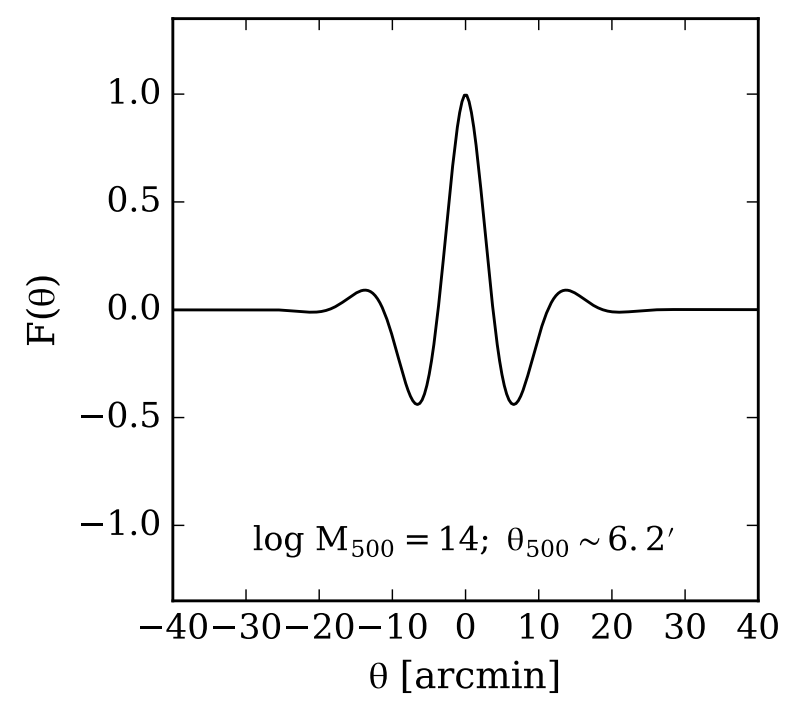

FIG. 1.- An example of the matched filter constructed for the tSZ analysis. Here a universal profile of Arnaud et al. (2010) is adopted as a spatial filter for a group of $\log M_{500} / \mathrm{M}_{\odot}=14$ and an angular radius $\theta_{500} \sim 6.2^{\prime}$.

$M_{500}$, used above is the halo mass within $R_{500}$. The halo masses and radii provided in the group catalogs are $M_{200}$ and $R_{200}$, respectively. To convert these quantities to the corresponding $M_{500}$ and $R_{500}$, we assume NFW profiles (Navarro et al. 1997) and concentration parameters as given by Neto et al. (2007).

\section{METHOD AND ANALYSIS}

\subsection{The matched filter technique}

Detecting the SZ signals physically related with a galaxy system is not trivial, as other effects, such as the primary CMB anisotropies, Galactic foreground, and other sources, can all contaminate the signals we want to obtain (see Section 4). Using a simple aperture photometry to extract the signals may thus lead to large uncertainties in the extracted signals (see e.g. Melin et al. 2006). To limit source confusions and background contamination, we employ the matched filter (MF) technique, first proposed for SZ analyses by Haehnelt \& Tegmark (1996), which is designed to maximize the signal-to-noise for a SZ source by imposing prior knowledge of the signals given the noise power spectra. For the case considered here, this means to optimally extract the tSZ signals from groups of galaxies, under the constraint of the power spectrum of the noise of the Planck maps. In practise, we closely follow Melin et al. (2005, 2006), who presented an extended and general formalism to extract signals from SZ surveys using the multi-filtering technique of Herranz et al. (2002). Such a MF technique has been applied in many recent analyses of the SZ effects in different surveys (e.g. Planck Collaboration V 2013; Planck Collaboration XI 2013; Planck Collaboration LIII 2017; Li et al. 2014; Le Brun et al. 2015).

In the MF approach, the Fourier transform of the filter that maximizes the signal-to-noise is given by:

$$
\hat{F}(\boldsymbol{k})=\left[\int \frac{\left|\hat{\tau}\left(\boldsymbol{k}^{\prime}\right) \hat{B}\left(\boldsymbol{k}^{\prime}\right)\right|^{2}}{P\left(k^{\prime}\right)} \frac{\mathrm{d}^{2} k^{\prime}}{(2 \pi)^{2}}\right]^{-1} \frac{\hat{\tau}(\boldsymbol{k}) \hat{B}(\boldsymbol{k})}{P(k)}
$$

where $\hat{\tau}(\boldsymbol{k})$ is the Fourier transform of the assumed spatial profile of groups, $\hat{B}(\boldsymbol{k})$ is the Fourier transform of a Gaussian beam function that mimics the convolution in Planck observation, and $P(k)$ is the noise power spectra. As the NILC $y$-map used here is already cleaned of the primary CMB anisotropies, $P(k)=P_{\text {noise }}$, where $P_{\text {noise }}$ is the power spectrum of the Planck noise map, as provided in the data release. The choice of the spatial filter function is not straightforward, and it can affect the integrated signals extracted. Indeed, using hydrodynamic simulations, Le Brun et al. (2015) found that the extracted tSZ signals can change significantly depending on the filter shape adopted. In our analysis, we adopt the universal pressure profile (UPP) given in Arnaud et al. (2010, hereafter A10), the form of which can be written as

$$
P(r)=A[E(z)]^{8 / 3} \mathcal{P}\left(r / R_{500}, M_{500}\right),
$$

where $E(z) \equiv H(z) / H_{0}, \mathcal{P}$ specifies the shape of the profile, and $A$ is an overall amplitude (see A10 for details). This profile was derived from a combination of X-ray observations of XMM-Newton REXCESS cluster sample (Böhringer et al. 2007) at $r \leq R_{500}$ and hydrodynamic simulations at larger radii. As a test, we have also used the spatial filter adopted in Le Brun et al. (2015) but did not find any significant changes in our results.

Figure 1 shows an example of the constructed filters, which assumes a group with $\log \left(M_{500} / \mathrm{M}_{\odot}\right)=14$ and an angular radius $\theta_{500} \sim 6.2^{\prime}$, and the universal pressure profile given above.

\subsection{Extracting the $t S Z$ signal}

Theoretically, the tSZ signal is characterized by a Compton $y$-parameter,

$$
y \equiv \frac{\sigma_{\mathrm{T}}}{m_{\mathrm{e}} c^{2}} \int P_{\mathrm{e}} \mathrm{d} l
$$

where $\sigma_{\mathrm{T}}$ is the Thompson cross-section, $m_{\mathrm{e}}$ the restmass of electron, $c$ the speed of light, $P_{\mathrm{e}}=n_{\mathrm{e}} k_{\mathrm{B}} T_{\mathrm{e}}$ the electron pressure, and the integration is over the line-ofsight to the observer.

The filters described above are then put at the group centers and 'matched' to the $y$-map to yield an estimate of the tSZ flux within $R_{500}, Y_{500}$ defined by

$$
d_{\mathrm{A}}(z)^{2} Y_{500}=\frac{\sigma_{\mathrm{T}}}{m_{\mathrm{e}} c^{2}} \int_{R_{500}} P_{\mathrm{e}} \mathrm{d} V,
$$

where $d_{\mathrm{A}}(z)$ is the angular diameter distance to a group at redshift $z$. Since $Y_{500}$ depends mainly on halo mass at a given $z$ and evolves with $z$ as $E^{2 / 3}(z)$, at a fixed halo mass, it is useful to define a new quantity,

$$
\tilde{Y}_{500} \equiv Y_{500} E^{-2 / 3}(z)\left(\frac{d_{\mathrm{A}}(z)}{500 \mathrm{Mpc}}\right)^{2},
$$

which is expected to be a function of only halo mass scaled to $z=0$, if the intrinsic tSZ flux is indeed only a function of mass.

To extract the SZ signals associated with galaxy groups from the observed $y$-map, a matched filter is put at each of all the groups in our group sample according to its halo mass and redshift. We then tune simultaneously the amplitudes of the filters for individual $M_{500}$ bins as listed in 


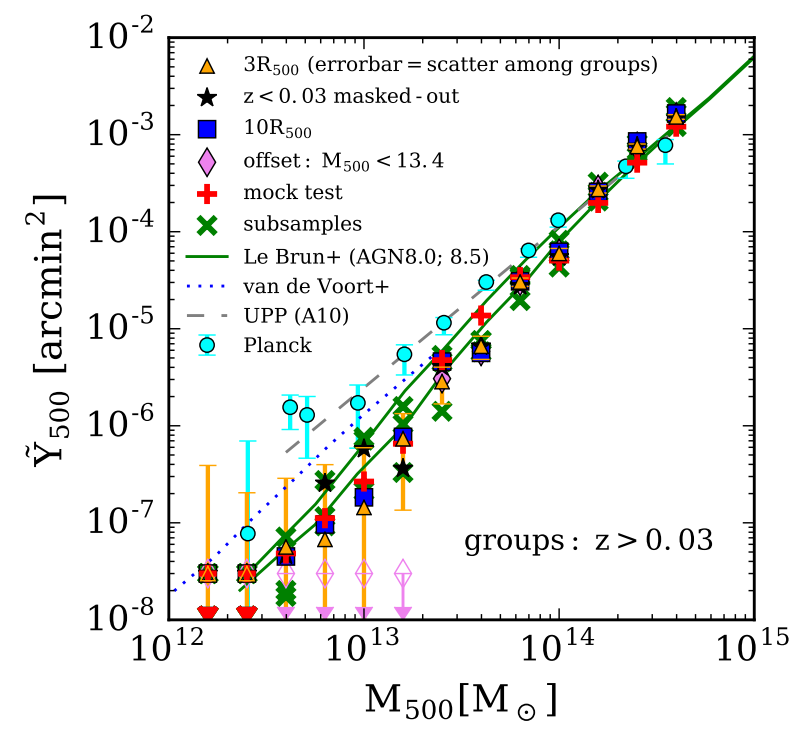

FIG. 2.- The $M_{500}-\tilde{Y}_{500}$ relations obtained by applying the matched filters, with shapes following that of Arnaud et al. (2010) (A10) truncated at $3 R_{500}$ (triangles) and $10 R_{500}$ (squares), respectively, and by masking out the pixels covered by groups at $z<0.03$ (stars). The case where the filters for groups with $\log \left(M_{500} / \mathrm{M}_{\odot}\right)<13.4$ are offset by $3 \theta_{500}$ is shown by diamonds. The red crosses plot the difference between the results from the coadded map and the added component (see text for details of this test). The green crosses show the results obtained from three independent sub-samples of total sample. The results are compared with those from Planck Collaboration XI (2013) (cyan dot), the universal pressure profile of A10 (UPP; dashed), Le Brun et al. (2015) (the two solid lines, with the upper one for their AGN8.0 and the lower one for AGN8.5), and van de Voort et al. (2016) (dotted line). The error bars indicate the scatters among the signals from individual systems in each mass bin. The unfilled symbols with downward arrows are used for cases where the tSZ fluxes are negative.

Table 1, assuming the amplitudes for all the groups in a given $M_{500}$ bin to be the same. The overall best match between the matched filters and the observed $y$-map is sought on the basis of the sum of the $\chi^{2}$ over all the pixels covered by the filters. The simultaneous matching of individual groups allows us to take into account the lineof-sight contributions from other halos, which is important, particularly for low-mass systems (see next section). For most of our analyses, we truncate the filter at $3 \theta_{500}$ and only use fluxes within it to estimate the integrated flux, where $\theta_{500}=R_{500} / d_{\mathrm{A}}$. Note that $R_{500} \sim 0.5 R_{200}$. As shown later in Section 4, truncation of the filters at $10 \theta_{500}$ leads to little change in our results. Finally, the mean flux within $R_{500}$ for groups in a given mass bin is estimated from the assumed spatial profile together with the amplitude obtained from the best match. The fluxes within $R_{500}$ for individual groups in a $M_{500}$ bin are also estimated by fixing the amplitudes of the matched filters of other mass bins to their best fitting values, while tuning the amplitudes of the filters for individual groups in the $M_{500}$ bin in question to achieve the best match.

\section{RESULTS}

\subsection{The $M_{500}-\tilde{Y}_{500}$ relation and the hot gas content}

Figure 2 shows our main result for the tSZ flux-halo mass relation. The values obtained from our fiducial

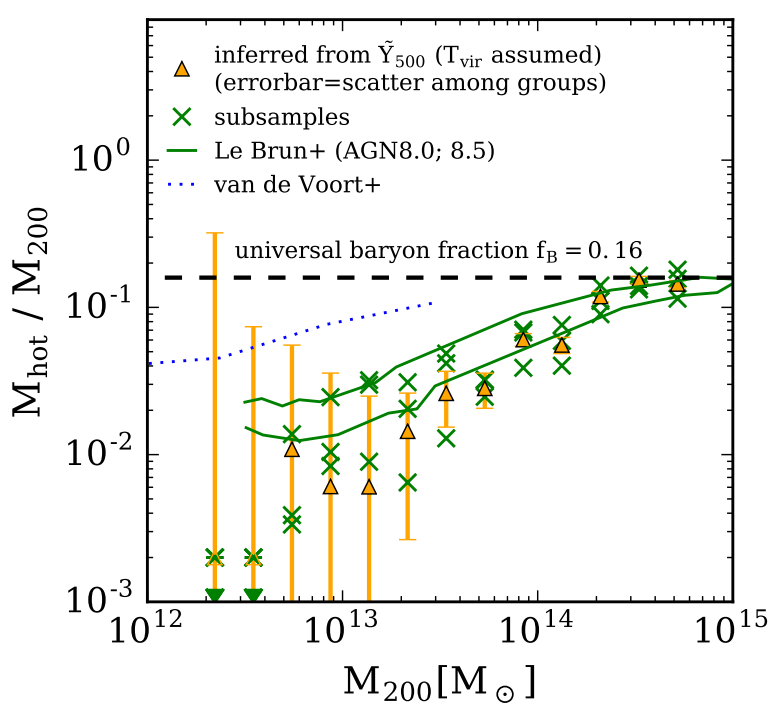

FIG. 3. - Hot gas mass fraction with respect to halo mass within $r_{200}$ as a function of halo mass inferred from $\tilde{Y}_{500}$ by assuming the virial temperature, compared with that from Le Brun et al. (2015) (solid; the upper for AGN8.0 and the lower for AGN8.5) and van de Voort et al. (2016) (dotted), which are based on hydrodynamic simulations. The gas content is lower than the universal baryon fraction of $f_{B}=0.16$ (dashed) in low-mass systems by a factor of up to $\sim 10$. Note that the gas mass ratio estimated is inversely proportional to a temperature assumed. The error bars indicate the scatters of the signals among individual systems in each mass bin. The crosses show the results obtained from three independent sub-samples. The unfilled symbols are used for those with the tSZ flux below zero.

TABLE 1

THE $M_{500}-\tilde{Y}_{500}$ RELATION $^{\mathrm{a}}$

\begin{tabular}{cccc}
\hline \hline $\log M_{500} / \mathrm{M}_{\odot}$ & $\begin{array}{c}\tilde{Y}_{500} \\
{\left[10^{-6} \mathrm{arcm}^{2}\right]}\end{array}$ & $\begin{array}{c}\text { Scatter } \\
{\left[10^{-6} \mathrm{arcm}^{2}\right]}\end{array}$ & No. of systems \\
\hline 12.3 & -0.0600 & 0.360 & 40,689 \\
12.5 & -0.0480 & 0.174 & 41,848 \\
12.7 & 0.0564 & 0.231 & 40,521 \\
12.9 & 0.0675 & 0.330 & 37,344 \\
13.1 & 0.144 & 0.450 & 32,063 \\
13.3 & 0.735 & 0.600 & 25,744 \\
13.5 & 2.85 & 1.17 & 19,020 \\
13.7 & 6.60 & 1.77 & 12,500 \\
13.9 & 30.3 & 3.00 & 6,203 \\
14.1 & 59.7 & 7.50 & 2,163 \\
14.3 & 273 & 22.8 & 484 \\
14.5 & 756 & 45.3 & 195 \\
14.7 & 1520 & 83.7 & 71 \\
\hline
\end{tabular}

a These data are presented in Fig. 2 by triangles.

$\mathrm{b}$ These are the scatters among individual systems in each mass bin.

sample are also given in Table 1 for reference. In all our analyses, we use only groups at $z>0.03$, to avoid the domination by a small number of nearby groups each covering a large number of pixels. The error-bars shown are each the $1 \sigma$ scatter among individual groups in the corresponding mass bin. For comparison, we also divide the total sample into three independent sub-samples that have about the same amount of sky coverage, and apply the MF method to them separately. The results are shown by the green crosses in the figure. As one can 
see, the scatter among these sub-samples is comparable to that among individual groups, indicating that the uncertainties are dominated by systematic effects.

Comparing the result obtained from the fiducial case, where the matched filters are truncated at $3 R_{500}$ with that obtained by using a larger truncation radius, $10 R_{500}$, shows that $3 R_{300}$ is sufficient to cover the signals from individual groups.

Because the hot gas halos of groups are extended and the observational beam size is relatively large, projection effects may contaminate the signals, particularly for small groups, even though our simultaneous matching of filters is supposed to eliminate such effects. We thus carry out a number of further tests to examine any possible residuals due to projection effects. In our first test, we remove all pixels covered by the groups at $z<0.03$, and the results are shown by stars in Fig. 2. As one can see, the signals for low-mass systems are changed relative to the fiducial case, suggesting that our results for low mass groups may still be affected by projection effects. In the second test, the filters for groups below a halo mass limit are shifted by a given amount with respect to the group centers. Thus, if the signals extracted for these groups were not associated with them but produced by larger structures, such a shift would not change the signals obtained for these groups. As an example, the diamonds in Fig. 2 show the result in which filters for groups with $\log M_{500} / \mathrm{M}_{\odot}<13.4$ are shifted randomly by $3 R_{500}$, while the filters for more massive groups are still located at the group centers. As one can see, while no significant change is seen for groups with $\log M_{500} / \mathrm{M}_{\odot}>13.4$, the signals for the lower mass groups are reduced. This suggests that the signals detected in the matched filters are associated with these low-mass groups. Finally, we make another test by adding to each group an artificial $y$-parameter profile which is given by the observed mean profile corresponding to its mass and redshift. The matched filter technique is then applied to the sum of this artificial map with the original map. The original signals are well recovered by the differences between the results obtained from the co-added map and the added component, as shown by the crosses in Fig. 2, demonstrating that our method can extract the signals we put in reliably.

Based on the test results presented above, we conclude that the results for groups with masses above $10^{13.5} \mathrm{M}_{\odot}$ are stable. For groups of lower masses, however, significant variations are still present from sample to sample.

Assuming virial temperatures, we estimate the hot gas contents of galaxy groups within $R_{200}$ from the integrated fluxes of $\tilde{Y}_{500}$. Here the NFW profile and the hot gas profile of A10 are used to convert quantities to the corresponding ones within $R_{200}$, and the virial temperature is defined as

$$
T_{\mathrm{vir}}=\frac{\mu m_{\mathrm{p}} G M_{200}}{2 k_{\mathrm{B}} R_{200}}
$$

where $\mu$ is the mean molecular weight, $m_{\mathrm{p}}$ the proton mass, and $k_{\mathrm{B}}$ the Boltzmann constant. The results obtained from the fiducial sample and the three subsamples are shown in Fig. 3. Here we see that the inferred hot gas contents of low-mass groups within $R_{200}$ are lower than the universal baryon fraction, shown by the horizontal line, by a factor of $\sim 10$. Even for groups with $M_{200} \sim 10^{14} \mathrm{M}_{\odot}$, the hot gas fraction is only about a half; only in the most massive groups (clusters) is the fraction close to unity. Such low hot gas contents in lowmass groups have important implications for theories of galaxy formation, as to be discussed in the following.

\subsection{Comparisons with earlier results and theoretical models}

Planck Collaboration XI (2013) (PCXI hereafter) used the same Planck data and a similar matched filter approach to extract the tSZ signals around locally brightest galaxies (LBGs) selected from the SDSS survey. An isolation criterion is adopted so that each LBG is the dominating one (in terms of luminosity) in its neighborhood, probably representing the central galaxy of a halo. Based on the mean relation between the stellar masses of central galaxies and the halo masses obtained from the semi-analytic galaxy formation model of Guo et al. (2013), a halo mass is assigned to each of the LBGs. The $\tilde{Y}_{500}-M_{500}$ relation obtained by PCXI is plotted in Fig. 2 as circles, and matches well the expectation of the UPP model of A10, shown by the dashed line. As one can see, our results are in good agreement with that of PCXI only for massive groups with $M_{500}>10^{14} \mathrm{M}_{\odot}$, but the amplitudes we obtain for groups of lower masses are much lower. Indeed, our $\tilde{Y}_{500}-M_{500}$ relation is very different from that given by the UPP model. We suspect that there are two factors that may cause the difference between our and PCXI results. First, we simultaneously match all groups in our sample, which takes into account the projection effects by larger halos along the line-ofsights of low-mass groups, while PCXI matches individual filters separately. In a test where we first subtracted the local flat backgrounds averaged over annulus between $\left[2 R_{200}, 3 R_{200}\right]$ around each group, and then matched individual filters and stacked the signals for groups of similar masses, we found that we can roughly recover the results of PCXI for low-mass halos, despite of the differences in other details between our method and theirs. This indicates that the contamination by other groups is not flat, and that it is important to match the filters to all groups simultaneously in order to correct for such projection effects.

Second, PCXI uses the mean relation between the central galaxy mass and halo mass to estimate halo mass, while our halo masses are estimated from our halo-based group finder. Given that the central galaxy mass increases only slowly with halo mass at $M_{200}>10^{13} \mathrm{M}_{\odot}$ (Yang et al. 2003), and the relation has significant amounts of scatter (e.g. Yang et al. 2008), binning based on central galaxy mass may mix halos of very different masses.

Greco et al. (2015) used a LBG sample similar to that used by PCXI, together with the Planck temperature aperture photometries, instead of the matched filter, to extract tSZ signals associated with the LBGs. They found that their results are consistent with the UPP model within the uncertainties of the data. It is unclear if the difference between their results and ours is produced by the different mass proxies used to bin the data or by the different methods used to extract the tSZ signals. Vikram et al. (2017) examined the cross-correlation be- 
tween groups in the catalog of Yang et al. (2007) and the Planck $y$-map, and found that two-halo terms dominate the signals around halos of $M_{200} \leq 10^{13-13.5} h^{-1} \mathrm{M}_{\odot}$. This is in qualitative agreement with our finding that the stacked signals for low-mass groups are dominated by projection effects.

We also compare our results with results from two hydrodynamic simulations. The first is that presented in Le Brun et al. (2015), who used the cosmo-OWLS suite of cosmological simulations (Le Brun et al. 2014), an extension of the OverWhelmingly Large Simulations (OWLS; Schaye et al. 2010), to model the tSZ effects. The simulation has a box size of $400 h^{-1} \mathrm{Mpc}$ on a side, and assumes cosmological parameters either from the WMAP7 or the Planck. Their fiducial runs include both stellar and AGN feedbacks. In Figs. 2 and 3, the predictions of two of their models are plotted as the two solid curves. The upper curves correspond to their AGN feedback model AGN8.0, which assumes that accreting black holes heat their surrounding gas to a temperature $\Delta T_{\text {heat }}=10^{8} \mathrm{~K}$, while the lower curves are for their AGN8.5, which assumes $\Delta T_{\text {heat }}=3 \times 10^{8} \mathrm{~K}$. Clearly, our results are in a good agreement with their results, particularly from that of the AGN8.5 run.

van de Voort et al. (2016) used a suite of cosmological zoom-in simulations from the Feedback In Realistic Environments (FIRE; Hopkins et al. 2014; Faucher-Giguère et al. 2015; Feldmann et al. 2016) project, to study the tSZ effects around halos with $M_{500}=10^{10}-10^{13} \mathrm{M}_{\odot}$. Sixteen and thirty six zoom-in simulations were run to $z=0$ and $z \sim 2$, respectively. In Figs. 2 and 3, we use straight lines to roughly represent their low- $z$ results. Here the universal profile of A10 is used to convert the predictions, which are integrated quantities within projected radius, to quantities within spheres needed in the comparison. It is seen that the predicted tSZ signals are much stronger than both our results and the simulations of Le Brun et al. (2015). We note, however, that the simulations used by van de Voort et al. (2016) do not include AGN feedback, which may be important for the halo mass range concerned here.

\section{SUMMARY AND CONCLUSION}

In this paper, we use the measurements of the thermal Sunyaev-Zel'dovich (tSZ) effect from the Planck NILC all-sky Compton parameter map, together with the group catalogs of Lim et al. (2017) to investigate the hot gas contents of galaxy groups. The catalogs contain a large number of uniformly selected groups with reliable halo mass estimates, which allows us to bin groups of similar halo masses to investigate the dependence of the tSZ effect on halo mass over a large mass range. We adopt the matched filter approach (Haehnelt \& Tegmark 1996; Herranz et al. 2002; Melin et al. 2005, 2006), which optimizes the signal-to-noise ratio by imposing prior knowledges of the expected signals, to extract the tSZ signals produced by galaxy groups from the map. We jointly match the filters to all groups to minimize projection effects.

We test the robustness of our method by retaining or eliminating pixels covered by local galaxy systems, by truncating the matched filters at different radii, by shifting the filters for low-mass groups, and by adding artificial signals to the observational map. We find that our method performs well in these tests. We also found that the background fluctuations around low-mass systems are significantly affected by projections of massive halos. Such a projection effect can lead to overestimation of the tSZ signals associated with low-mass groups if filters are not matched simultaneously to all groups.

We find that the integrated $y$-parameter and the hot gas content it implies are consistent with the predictions of the UPP model only for massive groups with masses above $10^{14} \mathrm{M}_{\odot}$, but much lower, by a factor of $\sim 10$, than the model prediction for low-mass groups. Our results are in conflict with the findings from some previous studies (e.g. Planck Collaboration XI 2013; Greco et al. 2015), which reported that their data are in agreement with the predictions of UPP model. The disagreement likely comes from the different treatments of projection effects and the different halo mass models used in these studies. The halo mass dependence we find is in good agreement with the predictions of a set of hydro simulations presented in Le Brun et al. (2015) that include strong AGN feedback, but the simulations of van de Voort et al. (2016), which include only supernova feedback, over-predict the hot gas contents in galaxy groups by a factor of 5 to 10 .

Since the integrated $y$-parameter is a measure of the thermal energy content of the hot halo gas, our results indicate that this energy content in low-mass groups is much lower than that expected from the universal baryon fraction in a hot halo at the virial temperature. This has important implications for galaxy formation and evolution. Since the total baryon fraction of stars and cold gas in galaxy groups and clusters is found to be well below the universal baryon fraction (e.g. Fukugita \& Peebles 2004), it has been speculated that the missing baryons may be in hot defused halos. However, if the low energy content found here is due to a low gas content in the hot phase, then hot gas halos cannot account for the missing baryons. Alternatively, baryons originally associated with galaxy groups may be heated and ejected by some processes. The agreement of our results with the predictions of the simulation results of Le Brun et al. (2015) suggests that strong AGN feedback may be able to provide such a process and to accommodate the observational results. Yet another possibility is that a large fraction of baryons may be in phases with temperatures much lower than the virial temperatures of the groups. In this case, the low thermal energy contents observed in low-mass halos are produced by the low gas temperature rather than by a reduced amount of gas. To distinguish the different possibilities, it is crucial to estimate the total mass in the warm-hot phase, so as to obtain a complete inventory of the baryons in low-mass halos. This can be done either through quasar absorption studies, or by investigating the kinetic SZ effect of galaxy groups, which depends on the electron density but not the temperature of the halo gas.

We thank Eiichiro Komatsu for helpful discussions. HJM acknowledges the support from NSF AST-1517528. This work is also supported by the China 973 Program (No. 2015CB857002) and the national science foundation of China (grant Nos. 11233005, 11621303, 11522324, 11421303, 11503065, 11673015), and South Africa Na- 
tional Research Foundation (grant no.105925).

\section{REFERENCES}

Arnaud, M., Pratt, G. W., Piffaretti, R., Böhringer, H., Croston, J. H., \& Pointecouteau, E. 2010, A\&A, 517, 92 1, 1, 3.1, 2 Battaglia, N., Bond, J. R., Pfrommer, C., \& Sievers, J. L. 2012, ApJ, 758, 741

Battaglia, N., Bond, J. R., Pfrommer, C., \& Sievers, J. L. 2013, ApJ, 777, 123

Böhringer, H., Schuecker, P., Pratt, G. W., et al. 2007, A\&A, 469, 3633.1

David, L. P., Jones, C., Forman, W., Vargas, I. M., \& Nulsen, P. 2006, ApJ, 653, 2071

Faucher-Giguère, C.-A., Hopkins, P. F., Kereš, D., Muratov A. L. Quataert E., \& Murray, N. 2015, MNRAS, 449, 9874.2

Feldmann, R., Hopkins, P. F., Quataert E., Faucher-Giguère, C.-A., \& Kereš, D. 2016, MNRAS, 458, L14 4.2

Fukugita, M., \& Peebles, P. J. E. 2004, ApJ, 616, 6435

Gastaldello, F., Buote, D. A., Humphrey, P. J., Zappacosta, L., Bullock, J. S., Brighenti, F., \& Mathews, W. G. 2007, ApJ, 669,1581

Greco, J. P., Hill, J. C., Spergel, D. N., \& Battaglia, N. 2015, ApJ, 808, 151 1, 4.2, 5

Guo, Q., White, S. D. M., Angulo, R. E., et al. 2013, MNRAS, $428,13514.2$

Haehnelt, M. G., \& Tegmark, M. 1996, MNRAS, 279, 545 1, 3.1,

Herranz, D., Sanz, J. L., Hobson, M. P., et al. 2002, MNRAS, $336,1057 \quad 1,3.1,5$

Hopkins, P. F., Kereš, D., Oñorbe, J., Faucher-Giguère, C.-A., Quataert E., Murray, N., \& Bullock, J. S. 2014, MNRAS, 445, $581 \quad 4.2$

Le Brun, A. M. C., McCarthy, I. G., Schaye, J., \& Ponman, T. J. 2014, MNRAS, 441, 1270 1, 4.2

Le Brun, A. M. C., McCarthy, I. G., \& Melin, J.-B. 2015, MNRAS, 451, 3868 1, 3.1, 3.1, 3.1, 2, 3, 4.2, 5

Li, M., Angulo, R. E., White, S. D. M., \& Jasche, J. 2014 MNRAS, 443, 23113.1

Lim, S. H., Mo, H. J., Lu, Y., Wang, H., \& Yang, X. 2017, MNRAS, 470, 2982 1, 2.2, 5

Ma, Y.-Z., Van Waerbeke, L., Hinshaw, G., et al. 2015, J. Cosmology Astropart. Phys., 9, 0461
McCarthy, I. G., Schaye, J., Ponman, T. J., et al. 2010, MNRAS, 406, 8221

Melin, J.-B., Bartlett, J. G., \& Delabrouille, J. 2005, A\&A, 429, $4171,3.1,5$

Melin, J.-B., Bartlett, J. G., \& Delabrouille, J. 2006, A\&A, 459, 341 1, 3.1, 5

Mo, H. J., van den Bosch, F. C., \& White, S. D. M. 2010, Galaxy Formation and Evolution, Cambridge University Press, New York, NY 1

Navarro, J. F., Frenk, C. S., \& White, S. D. M. 1997, ApJ, 490, 4932.2

Neto, A. F., Gao, L., Cole, S., et al. 2007, MNRAS, 381, 14502.2

Planck Collaboration I. 2011, A\&A, 536, 12.1

Planck Collaboration V. 2013, A\&A, 550, 131 1, 3.1

Planck Collaboration XI. 2013, A\&A, 557, 52 1, 3.1, 2, 4.2, 5

Planck Collaboration XIII. 2016, A\&A, 594, 131

Planck Collaboration XXII. 2016, A\&A, 594, 112.1

Planck Collaboration LIII. 2017, [arXiv:astro-ph/1707.00132] 3.1

Pratt, G. W., Croston, J. H., Arnaud, M., \& Böhringer, H. 2009, A\&A, 498, 3611

Remazeilles, M., Delabrouille, J., Cardoso, J.-F., et al. 2011, MNRAS, 418, 4672.1

Schaye, J., Dalla Vecchia, C., Booth, C. M., et al. 2010, MNRAS, $402,1536 \quad 4.2$

Sun, M., Voit, G. M., Donahue, M., Jones, C., Forman W., \& Vikhlinin, A. 2009, ApJ, 693, 11421

Sunyaev, R. A., \& Zeldovich, Y. B. 1972, Comments on Astrophysics and Space Physics, 4, 1731

Tauber, J. A., Mandolesi, N., Puget, J., et al. 2010, A\&A, 520, 1 2.1

van de Voort, F., Quataert, E., Hopkins, P. F., et al. 2016, MNRAS, 463, 4533 2, 3, 4.2, 5

Vikram V., Lidz, A., \& Jain, B. 2017, MNRAS, 467, 2315 1, 4.2

Yang, X., Mo, H. J., \& van den Bosch, F. C. 2003, MNRAS, 339, $1057 \quad 4.2$

Yang, X., Mo, H. J., van den Bosch, F. C., \& Jing, Y. P. 2005, MNRAS, 356, $1293 \quad 1,2.2$

Yang, X., Mo, H. J., van den Bosch, F. C., Pasquali, A., Li, C., \& Barden, M. 2007, ApJ, 671, 153 1, 2.2, 4.2

Yang, X., Mo, H. J., \& van den Bosch, F. C. 2008, MNRAS, 676, $248 \quad 4.2$ 